$$
\text { DOE/ER/45089--T! }
$$

Final Technical Report on DOE Sponsored Project $n^{2+\infty}$ "Condensed Matter Research Using the UCSB FEL" DOE/ER/45089-TI DE.FG03-84ER45089 DE92 040560<smiles>C1#[Si][Si]C1</smiles>

8

(1)

$\cos$ $5 / 1 . / 84-1 / 31 / 92$

\title{
I. Historical Perspective
}

The University of California Santa Barbara (UCSB) Free Electron Laser (FEL) project was initiated in 1981 by the ONR and the AFOSR to test the idea of using an electrostatic accelerator in a recirculating beam mode to produce high power, continuously tunable, coherent far infrared (FIR) radiation. This was a proof of-concept development project and contained no provisions (space, instrumentation or personnel) for utilizing the FIR radiation for scientific application; e.g. condensed matter research.

In the anticipation of a successful demonstration of the operation of the UCSB FEL, we began in 1983 to seek support from various governmental agencies for a FIR users program aimed at condensed matter science. It is to the credit of Tom Kitchens and Lou Ianello that funding was obtained in 1984 to begin the research reviewed here under the aegis of the DOE.

Although the DOE-sponsored program began on May 1, 1984, it was understood that a very considerable effort would have to be directed towards preparatory work, e.g. the design and construction (or purchase) of FIR characterization and detection equipment. This included a FIR Fabrey Perot interferometer, a $\mathrm{CO}_{2}$ " alcohol laser and an InSb Putley detector. Furthermore, a sizable fraction of our initial effort was spent in assisting the FEL development group in characterization studies such as short and long term stability, pulse shape as a function of electron beam recovery and frequency tunability. These studies began shortly after the first operation of the FEL in August of 1984 until the late spring of 1985 when the conversion of the accelerator from a $3 \mathrm{MeV}$ too a $6 \mathrm{MeV}$ machine was made.

We postponed initiating our research studies until the accelerator conversion was com- 


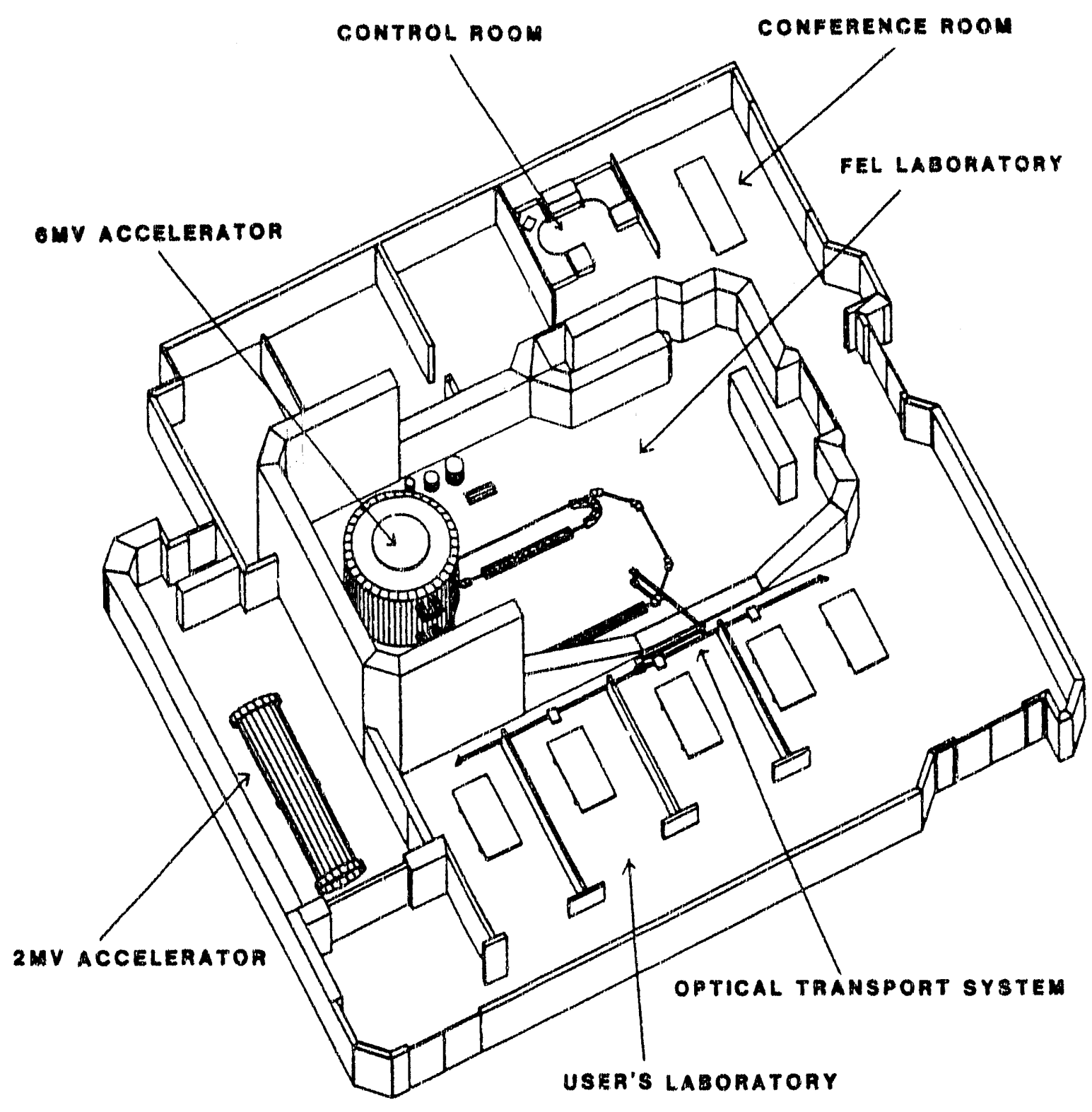

THE CENTER FOR FREE ELECTRON LASER STUDIES UNIVERSITY OF CALIFORNIA, SANTA BARBARA 


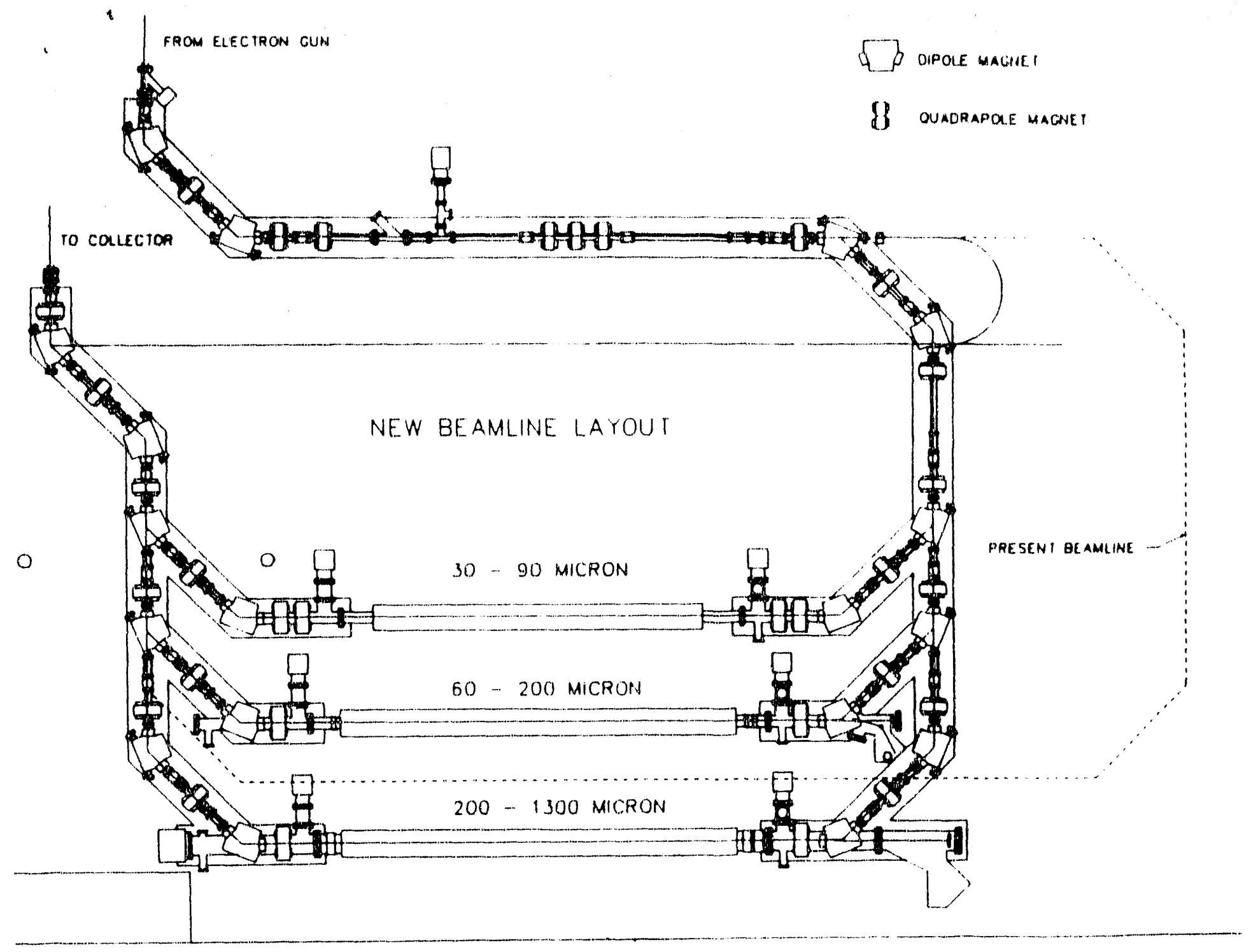

\begin{tabular}{|c|c|c|c|}
\hline \multicolumn{4}{|c|}{ DESIGN PARAMETERS OF UCSB FELS } \\
\hline & MM-WAVE FEL & FIR FEL & MID-IR FEL \\
\hline Wavelength & $1.34 \mathrm{~mm}-193 \mu \mathrm{m}$ & $435-63 \mu \mathrm{m}$ & $64-30 \mu \mathrm{m}$ \\
Power" & $4-37 \mathrm{KW}$ & $4-14 \mathrm{KW}$ & $4-8 \mathrm{KW}$ \\
w/cavity dump & $230-740 \mathrm{KW}$ & $220-290 \mathrm{KW}$ & $130-160 \mathrm{KW}$ \\
Pulse Length" & $30 \mu \mathrm{s}$ & $30 \mu \mathrm{s}$ & $30 \mu \mathrm{s}$ \\
w/cavity dump & $37 \mathrm{~ns}$ & $37 \mathrm{~ns}$ & $37 \mathrm{~ns}$ \\
Beam Energy & $2.0-6.0 \mathrm{MEV}$ & $2.0-6.0 \mathrm{MEV}$ & $4.0-6.0 \mathrm{MEV}$ \\
Beam Current & $2.0 \mathrm{~A}$ & $2.0 \mathrm{~A}$ & $2.0 \mathrm{~A}$ \\
\hline
\end{tabular}

* Design values assume operation with terminal stabilizer

$\dagger$ Values assume coupling out of entire intra-cavity power 
plete in 1985. However, we used this time to develop an optical (magnon or phonon) side-band spectrometer and a nanosecond FIR switch. The former became an important component in our research in later years and the latter a powerful tool in the study of excitations with nanosecond or shorter lifetimes. (Details of these two projects were provided in early progress reports.)

For a good part of 1986, the UCSB FEL operated on an almost continuous basis, with time equally shared by the user research and the development efforts. Since all of our application work had to be carried out by remote control inside the shielded accelerator laboratory, and there was little space for optical benches, lasers and monochrometers, we decided to first do the simplest of experiments. This involved doing linear spectroscopy (demonstrating the ability of the FEL to be tuned through a resonance) and then a saturation experiment (to demonstrate the higher power available from the FEL than from the other sources.

\section{Initial FIR Research}

The first application of any FEL to condensed matter research in the world was our studies of the magnetic polariton in antiferomagnetic $\mathrm{FeF}_{2}$ and the impurity mocies associated with it arising from very small concentrations of $\mathrm{Mn}^{2+}(40 \mathrm{ppm})$. The results of the initial experiments are shown in Figs. 1 and 2 and have been detailed in the literature and a previous yearly progress report.

\section{Users Facility Laboratory}

It became clear almost immediately that it would be impossible to continue the user program without a separate contiguous laboratory into which th? FIR would be transported and to have several output ports for the different users. Fortunately, we were able to obtain funding from the ONR-managed, SDIO-FEL Biomedical and Material Sciences Research Program to build a Users Facility Laboratory and FIR beam transport system. 
Fig 1

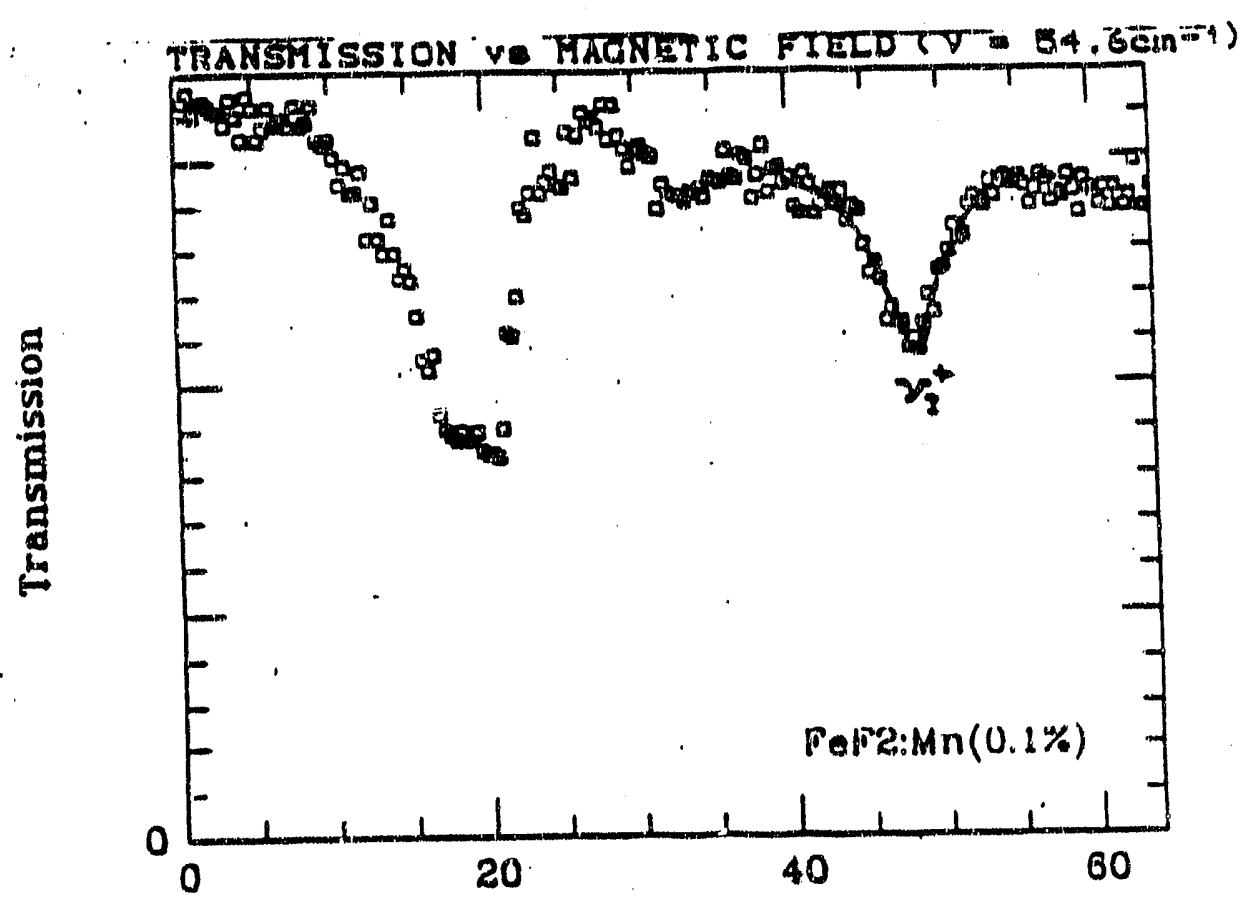

Fig 2

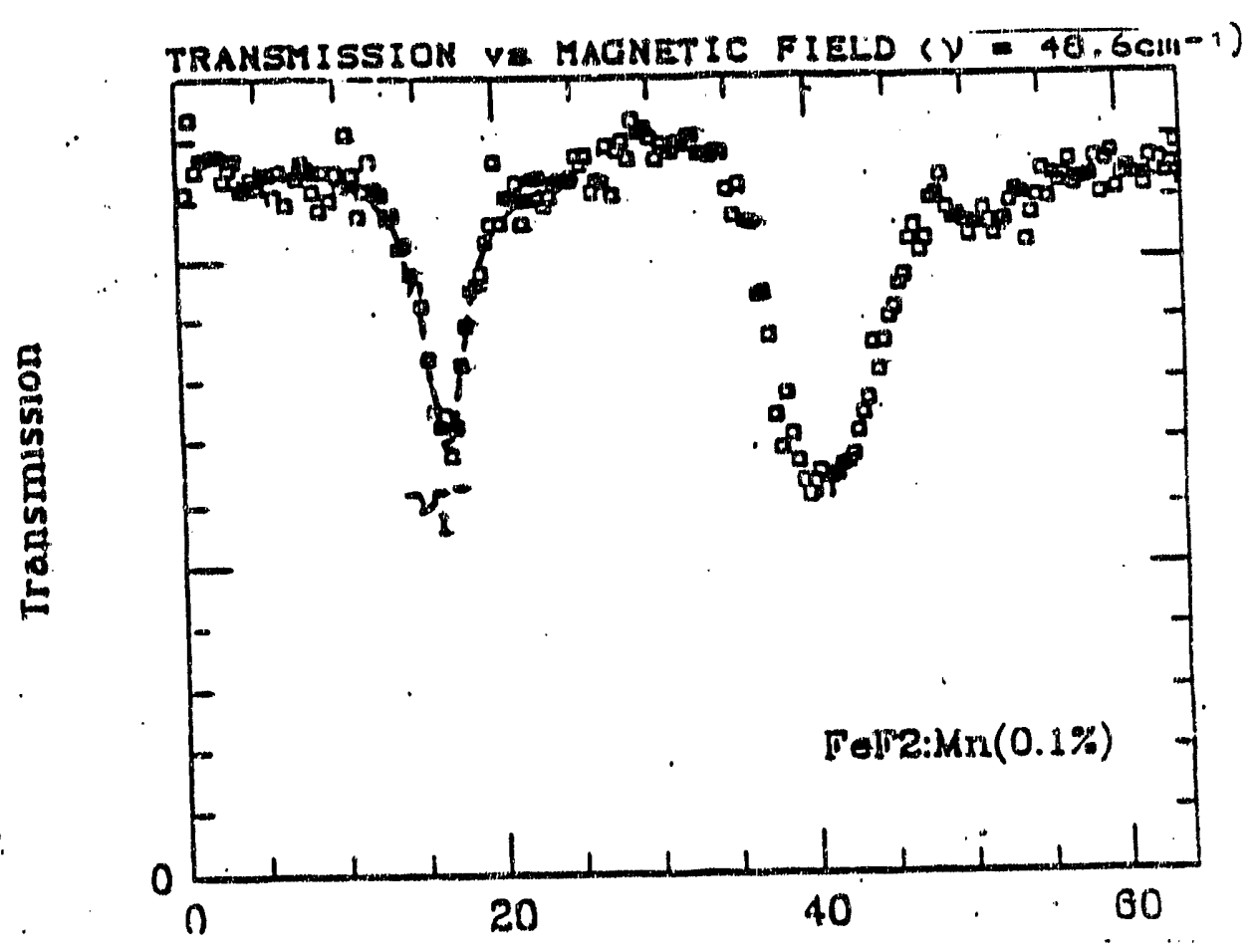

The host (AFMR) and Mn impurity modes vs magnetic fleld ( $1 \mathrm{n} \mathrm{kOe}$ ) at two frequenctes; a) $54.6 \mathrm{~cm}^{-1}$ and b) $48.6 \mathrm{~cm}^{-1}$. The dffference in the linewidths of $v_{I}^{+}$and $v_{I}^{-}$in these two cases is explained in the text. 
This laboratory was completed in December of 1986 and was equipped with $\$ 500,000$ of specialized laser, cryostats, magnets, detectors and auxiliary electronics for signal processing.

The ONR-SDIO contract also provided operating furds for the use and maintenance of the FEL in this user support role including technical staff and administration. We note this because neither the main FEL development effort or the DOE-sponsored program had any provision in them for providing these necessary functions. 'Thus the laboratory's capabilities became well known to other scientists in this ccuntry as well as abroad, the DOE-sponsored program was amplified by the collaborations we were able to establish through this broad support.

\section{Semiconductor Research (1987-1992)}

By far, the principal application of the UCSB FEL has been to research in modern semiconductors. The reasons for this are primarily two-fold: 1) a large number of the

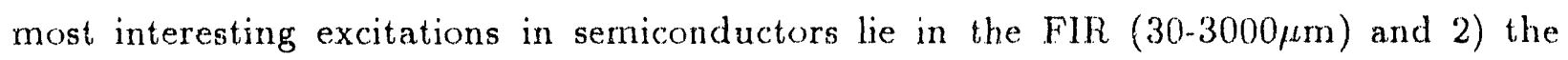
lifetimes of many of these excitations relatively long $(\tau>100 \mathrm{psec})$, making possible nonlinear/nonequilibrium studies at power levels easily cbtainable with the UCSB FEL.

Beginning in 1987, a series of experiments were performed on shallow donors in $n$ GaAs and on lower dimensional electron sy'stems such as GaAs/GaAlAs heterostructures to explore their nonlinear/nonequi ibrium properties. The electronic excitations include intersubband, cyclotron and plasmon resonances, all of which are in the FIR and are also electric or magnetic field tunable. They had been thoroughly investigated by linear spectroscopy.

Specific semiconductor FIR studies include:

1) Saturation of Shallow Impurity Transitions in n-GaAs. The long-pulse, high power of the FEL made possible steady-state conditions, heretofore not achievable with TEA. 
$\mathrm{CO}_{2}$ lasers. The most important result was the demonstration that a three-level model was necessary to describe the saturation behavior. The fits to the model yield the ionization probability of the $2 \mathrm{p}^{+}$state, the relaxation rate for the $2 \mathrm{p}^{+} \rightarrow$ ls transition and the recombination time of the free carriers. Later measurements at other frequencies and fields, combined with our short-pulse ( $\leq 10 \mathrm{nsec}) \mathrm{Nd}$ :YAG laser-semiconductor switch allowed measurements of the time evolution of the photoconductivity, from which a direct measurement of the $2 \mathrm{p}^{+} \rightarrow$ Is decay time $(\tau=15 \mathrm{nsec})$ was made. In these later studies both photoconductance as well as photo-Hall voltages were measured in an effort to distinguish between impurity hopping conduction and conduction band transport; however, the results showed the mobility and electron concentration were too large to account for impurity tunneling or hopping.

2) High Power Studies of Cyclotron Resonance in n-GaAs. Complete saturation in the $\mathrm{N}=0$ to $\mathrm{N}=1$ Landau level cyclotron resonance (CR) was observed. The ( $R$ was accompanied by an increasing shift to higher magnetic fields with increasing FIR power, indicative of a non-equal spacing between Landau Levels with $N>1$. Similar studies were made on In $\mathrm{P}, \mathrm{InSb}$ and 2 -dimensional heterojunctions.

3) Bistability and Conductance Oscillations in n-GaAs. When biased near but below the breakdown threshold, it was discovered that the conductance switches from a high to a low conductance state with alternate high-power FEL pulses. The sequence repeals itself, ad infinitum. We believe this to be an example of a nonequilibrium phase transition.

4) Nonlinear/Non Equilibrium Studies of Heterostructures. Plasmon excitations of the two-dimensional electron gas (2-DEG) in a GaAs/AlGa heterostructure have been studied with the UCSB FEL using a grating-coupler. An anomolous photovoltaic response is found in the nonlinear regime where saturation behavior is observed. When the same structure is biased with a negative gate voltage, the $2-\mathrm{DEG}$ is confined to one-dimensional channels; hence crnssover from 2-DEG to 1-DEG is observed. The excitations in the 1-DEG region 
are highly nonlinear and are easily saturated with the FEL.

\section{Iwo Photon Experiments}

There are a class of experiments which involve (strong) excitation of a system at one frequency and (weak) probing its response at another one - so called "two-photon" or "two-color" studies. Although a number of such experiments have been executed in the visible or IR region, no studies have been made involving FIR photons.

1) $\mathrm{Er}^{3+} \mathrm{LaF}_{3}$. The ${ }^{4} \mathrm{I}+{ }_{15 / 2}(2)$ state in $\mathrm{Er}_{\mathrm{LaF}} \mathrm{LaF}_{3}$ was excited by $52 \mathrm{~cm}^{-1}$ radiation from the FEL. Detection of the nonequilibrium population of this state was attempted, using an optical boxcar method, by which electrons in the ${ }^{4}{ }_{15 / 2}(2)$ were excited to the ${ }^{4} I+{ }_{15 / 2}(1)$ level and then the time integrated fluorescence measured. The results showed that the ${ }^{4} \mathrm{I}+{ }_{15 / 2}(2)$ state decay very rapidly with a value of $\tau \simeq 10 \mathrm{psec}$.

2) n-GaAs. The decay of the shallow-donor level $2 \mathrm{p}^{+}$can be probed by varying the time between the FEL pump pulse $\left(1 \Delta \rightarrow 2 \mathrm{p}^{+}\right)$and the probe pulse $\left(2 \mathrm{p}^{+} \rightarrow\right.$ conduction band). Preliminary two-FIR photon studies of this system were in progress on the last year of the grant.

3) Frequency, Temporal and Spatial Evolution of Magnons in MuF 2 . The dy namics of large wave vector magnons in antiferomagnetic $\mathrm{MuF}_{2}$ were studied using a FIR (pump) and visible (probe) laser technique. One-magnon excitation were generated by pumping the two-magnon band and then optically detected via the frequency-selective, anti-Stokes sideband of the E1 exiton. With this method, we were able to obtain the frequency, temporal and spatial evolution of the initial, nonequilibrium distribution of large $\vec{k}$ magnons. The most important results appear to be the very short lifetimes of the two-magnon states, the time constants for the energy diffusion of the one-magnon states ( $\sim 1 \mu \mathrm{sec})$ and the decay of magnons into phonons in times of the order of $5 \mu \mathrm{sec}$. No evidence for spatial diffusion of one-magnon states was found for times $t \geq 40$ nsec. 


\section{Summary}

The DOE-supported project provided the initial impetus for use of a FEL for condensed matter science. The program at UCSB, with major support from the ONR via the SDIO, developed into a model user program for othor FELs throughout the world. Exploratory experiments in a number of areas have demonstrated the utility of the UCSB FEL for basic research in condensed matter science. The decreasing support from DOE in the later years of this grant precluded the completion of a number of projects but it did provide for continuity in the work of several graduate students and post-doctorals.

\section{Personnel}

The following individuals have received all or part of their salaried support from this grant since its inception.

Graduate Students

Joseph Spector; Ph.D. thesis

Jann Kaminski; Ph.D. thesis

Abbas Nikroo; Ph.D. thesis

Post Doctorals

A.R. King

J. Kaminski

M. Warden

Secretary

Y. Schiada 
UCSB Center for Free Electron Laser Studies

\section{PUBLICATIONS}

"Free Electron Laser-Far Infrared Study of $F e F_{2}: M n$ ", J. Spector, J. Kaminski and V. Jaccarino, Solid State Communications, Vol. 6ㄴ, No. 12, pp. 1093-1095, 1987.

"Inhibition of Nucleic Acid Synthesis in Cells Exposed to 200 Micrometer Radiation. from the Free Electron Laser", M.W. Berns and W. Bewley, Photochemistry and Photobiology, Vol. 46, No. 2, pp. 165-167, 1987.

"High Power Non-Linear Magnetophotoconductivity in $n-$ GaAs Using The UCSB Free Electron Laser", J. Kaminski, J. Spector, W. Prettl and M. Weispfenning, International Journal of Infrared and Millimeter Waves, Vol. 9, pp. 745, 1988.

"Free-electron Laser Study of the Nonlinear Magnetophotoconductivity in $n-G a A s$ ", J. Kaminski, J. Spector, W. Prettl and M. Weispfenning, Appl. Phys. Lett. 52(3), pp. $233,1988$.

"Inpurity Hopping Conductivity in GaAs : Si Induced by FIR Radiation of a FEL", J. Kaminski, J. Spector, C.T. Foxon, A.C. Labrujere, T.O. Klaassen, W. Th. Wenckebach, 19th International Conference on the Physics of Semiconductors, Warsaw, Poland, August 15-19, 1988.

"A New Spectroscopy? Spin-Tunneling in Heme Proteins", R.H. Austin, B.S. Gerstman, P.A. Mansky and M.W. Roberson, Biophysical Journal, Vol. 53, pp. 260a, 1988.

"Proposal for the Direct Electromagnetic Generation of Coherent Terahertz Acoustic Phonons in Semiconductors Superlattices at the University of California, Santa Barbara, Far-Infrared Free Electron Laser Facility", T.E. Wilson, J. Opt. Soc. Am. B. Vol. 6, pp. 1.058, 1989.

"Material Applications of the Far-Infrared Free Electron Lasers to Condensed Matter Physics", J.D. Simon, J.E. Crowell. J.H. Weare and D.R. Miller, Vol. 6, Opt. Soc. Am. B, pp. 1035, 1989.

"Applications of Far Infrared Free Electron Lasers to Condensed Matter Physics", W.M. Dennis, Vol. ㅁ, J. Opt. Soc. Am. B, pp. 1045, 1989.

"Tunable Far-infrared Photovoltaic Response in Semiconductor Field-effect Devices", E. Batke, J. Kaminski, J.P. Kotthaus and J. Spector, Appl. Phys. Lett. 54(2), pp. 131, 1989.

"Far-infrared Photo-Hall Experiments on GaAs : Si", J. Kaminski, J. Spector, C.T. Foxon, T.O. Klaassen and W.Th. Wenckebach, J. Opt. Soc. Am. B/Vol 6, No. 5, 1989.

"Enhancement of Ligand Binding to Myoglobin by Far-infrared Ex itation from a FreeElectron Laser", B. Gerstman, M. Robertson and R. Austin, J. Opt. Soc. Am. B/Vol. 6, No. 5, 1989.

"Far-infrared Perturbation of Reaction Rates in Myoglobin at Low Temperatures", R. H. Austin, M.W. Roberson and P. Mansky, Physical Review Letters, Vol. 62, Number 1, pp. $1912,1989$.

"Far-infrared Cavity Dump Coupling of the UC Santa Barbara Free-Electrom Laser", J.P. Kaminski, J.S. Spector, C.L. Felix, D.P. Enyeart, D.T. White and G. Ramian, Appl. Phys. Lett. underbar 57(26) pp. 2770, 1990.

"Ballistic Propagation of Magnons", W. Grill and W.M. Yen, International Conference on Magnetism, Paris (1988), Journal of Luminescence, v45, n1-6, pp. 130-134, 1990. 
"Graded Potential Wells with Quasi-Uniform Charge Distribution", A. Wixforth et al, Surface Science 228, pp. 489-492, 1990.

"Demonstration and Applications of a FIR Cavity Dump Output Coupler at the UC Santa Barbara FEL Facility", J.P. Kaminski, D. Enyeart and D. White, Proceding of IRMM, 1990 .

"Current Applications Using the UCSB Free Electron Laser", J. Kaminski, Nuclear Instruments and Methods in Physics Research A296, p1. 784-786, 1990.

"Free Electron Laser Irradiation at $200 \mu \mathrm{m}$ Affects DNA Synthesis in Living Cells", M.W. Berns, W. Bewley, C.H. Sun and Pamela Templin, Proc. Natl. Acad. Sci., Vol. 87, pp. 2810-2812, 1990 .

"Generation of Subnanosecond High Power Far Infrared Pulses Using a FEL Pumped Passive Resonator", J. Burghoorn, J.P. Kaminski, R.C. Strijbos, T.O. Ḱlaassen, W. Th. Wenckebach, Journal of Opt. Soc. of Am. B- 1991.

"Narrow-Bandwidth Operation of a Free-Electron Laser Enforced Laser Enforced by Seeding", A. Amir, J.F. Knox-Scith and M. Warden, Physical Review Letters, Vol. 66, Number 1, pp. 29, 1991.

"Frequency, Temporal and Spatial Evolution of Large $\vec{k}$ Magnons in Antiferromagnetic $M n F_{2}$ ", A. Nikroo and V. Jaccarino, Submitted to the International Conference of Magnetism, Edinburgh, Scotland, 2-6 September, 1991.

"Nonlinear Far-infrared Photoacoustic Magnetospectroscopy of $n-G$ a As at Low Tenperatures", A. Schilz, L. Huber, W. Pretti and J. Kaminski, Appl. Phys. Lett. 60(19), pp. 2394, 1992.

"Saturation Spectroscopy of Carriers in Semiconductor Multiple-Quantum-Well Structures", R. Ranganathan, J. Kaminski, W.J. Li, P. Cheng and B.D. McCombe, (to be published).

"Far Infrared Perturbation of Electron Tunneling in Reaction Centers?", R.H. Austin and M.K. Hong, (to be published).

"FIR Saturation Spectroscopy of the Mn Inpurity Mode in Antiferromagnet $F c F_{2}$ ", J. Kaminski and V. Jaccarino (to be published).

"Generation of Subnanosecond High Power Far Infrared Pulses Using a FEL Pumped Passive Resonator", J. Burghoorn, J.P. Kaminski, R.C. Strijbos, T.O. Klaassen and W. Th. Wenckebach, (to be published). 
UCSB Center for Free Electron Laser Studies

\section{MEETING TALKS}

"Solid State FIR Spectroscopy with the UCSB Free Electron Laser", J. Spector, J. Kaminski, D. Gregoire, J. Hu and V. Jaccarino. Presented at the American Physical Society Meeting, Las Vegas, March 31-April 4, 1986 (contributed).

"First Application of the UCSB FEL to Condensed Matter Research", V. Jaccarino. Presented at International Conference on Quantum Electronics, San Francisco, June 9-13, 1986 (invited).

"Free Electron Laser Study of the Nonlinear Magnetophotoconductivity in n-GaAs", Jann Kaminski. Presented at the International Conference on Millimeter and sub-Millimeter Waves", Lake Buena Vista, Florida, December 13-19, 1987 (invited).

"Current Applications Using the UCSB Free Electron Laser", Jann Kaminski, Proc. of the 11th Int'l Free Electron Laser Conference, August 1989, Naples, Florida, 1989.

\section{COLLOQUIA}

"Condensed Matter Science Using the UCSB FEL" Presented by V. Jaccarino at the University of British Columbia (3/87); University of Leiden, The Netherlands (4/87); AT \& T Bell Labs (9/87); University of California, San Diego (9/87); University of California, Irvine (10/87; University of California, Riverside (1/88); General Motors Research Laboratory, Warren, Mich. (2/88). 
UCSB Center for Free Electron Laser Studies

\section{DISSERTATIONS}

"Design and Performance of Undulators at the UCSB Free-Electron Laser", Ren-Chau James Hu, March 1986.

"The First Applications of the University of California of California, Janta Barbara, Free Electron Laser to Condensed Matter Research". Joseph S. Snector, Febr "rary 1088.

"Semiconductor Studies Using the UCSB Free-Electron Laser". Jann Paul Kaninski, February 1989.

"Evaluation of a 4 mim Period LCSB Micro-Undulator", Kevin Phillip Paulson, September 1990.

"Injection Seeding of an Electrostatic Acceleratior Driven FEL", John Finn KnoxSeith, October 1990.

"Impurity Associated Magnetic Modes in Antiferromagnets". David William Donnelly, Nov. 1990.

"The Frequency, Temporal, and Spatial Evolution of Large Wavevector Magons in $M F_{2} "$, Abbas Nikroo, Nov, 1990.

* DOE supported

\section{DISCLAIMER}

This report was prepared as an account of wrork sponsores by an agency of the United States

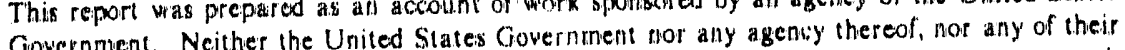
employess, makes any warranty, express or implied, or assumes any legal liability or responkibility for the accuracy, completeness, or usefulmess of any information, apparalus, product, or ptocess disclosed, of represents that its use would not infrimge privately owned rights. Refer. ence herein to any specific commercial product, process, or service by trade name, trademark. masufacturer, or otherwise does not necessarily constitute or imply its endorsement, recom. mendation, or faworing by the United States Government or any agency thereof. The views and opinions of autiors expressed herein do not necesssaribly state or reflect those of the United States Government or any agency thereof. 

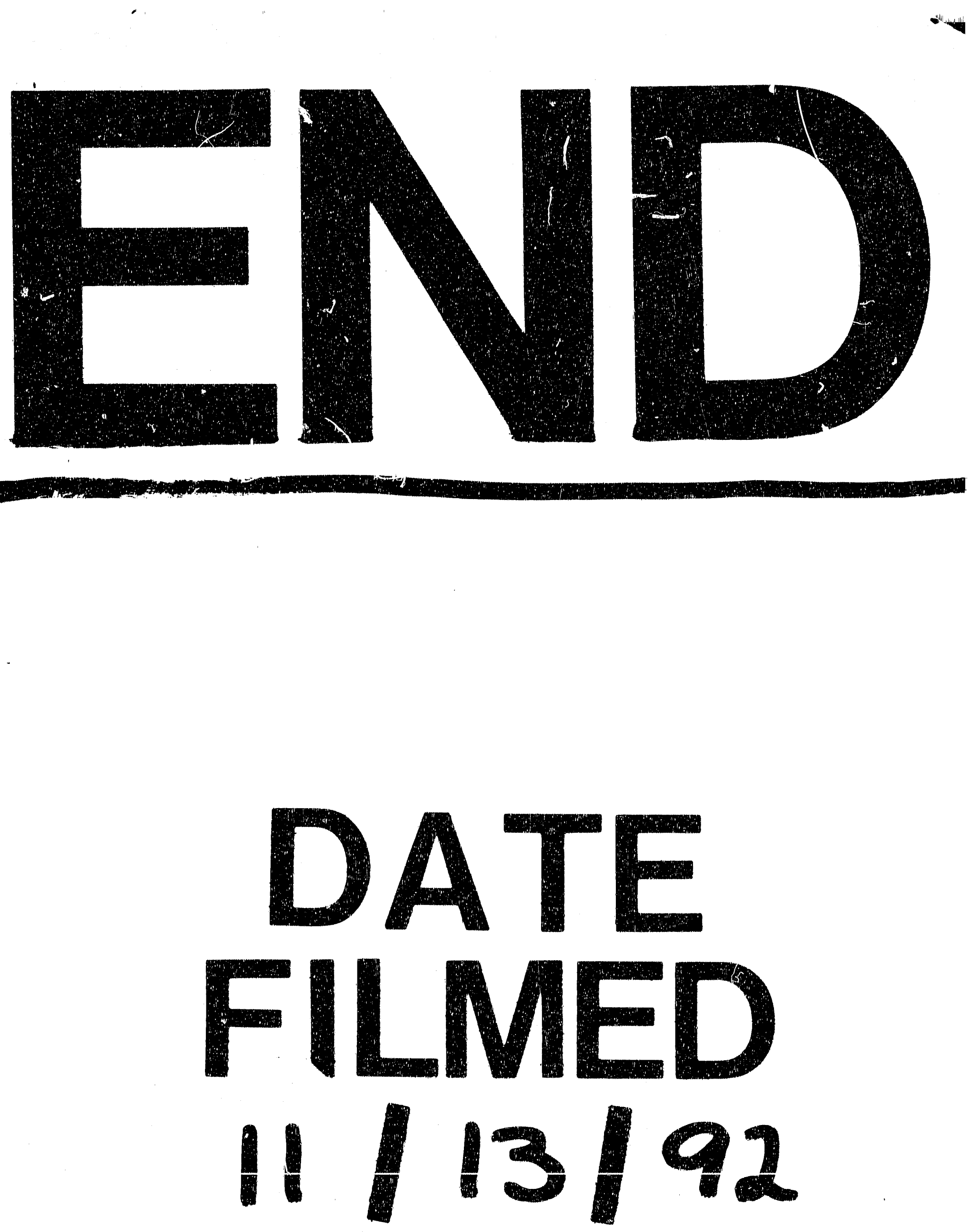


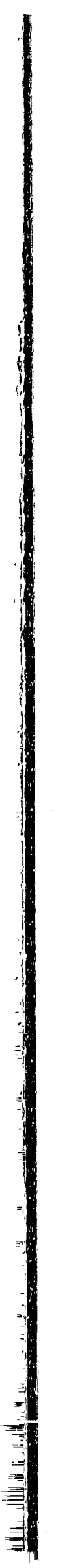

